\title{
Algebra for Tree Languages ${ }^{\star}$
}

\author{
Mikołaj Bojańczyk \\ Institute of Informatics, Warsaw University, Poland \\ bojan@mimuw.edu.pl
}

There are at least as many interesting classes of regular tree languages as there are of regular word languages. However, much less is known about the former ones. In particular, very few effective characterizations of tree language classes are known. Since for words most known characterizations are obtained using algebra, it seems to be a good idea to look for an algebra for tree languages. I will talk about one such attempt, which is called forest algebra. (Other frameworks in the literature include pre-clones of Ésik and Weil or tree algebra of Wilke. Another approach is to forget about algebra and study the structure of a tree automaton.)

In the algebraic theory of word languages, elements of a monoid or semigroup are used to represent words. In a forest algebra, there are two sorts. The first sort represents forests, and the second sort represents contexts (forests with a hole). The operations of forest algebra include concatenation of forests (concatenating a forest of $n$ trees with a forest of $m$ trees gives a forest with $n+m$ trees) and composition of contexts (composing a context where the hole is at depth $n$ with a context where the hole is at depth $m$ gives a context where the hole is at depth $n+m)$.

I will talk about some recent work, which tries to relate the algebraic structure of a forest algebra with the type of tree languages that this algebra recognizes. A very simple example of this kind says that if the algebra has commutative concatenation, then the languages it recognizes are invariant under swapping siblings. Of course, the theory contains some non-trivial results.

A big open problem in the field is finding an effective characterization of firstorder logic. One of the reasons why forest algebra was developed is the hope that there is some structural property of forest algebra - something like aperiodicity for monoids - that corresponds to first-order definability of a tree language.

\footnotetext{
^ Joint work with: Luc Segoufin, Howard Straubing and Igor Walukiewicz. 